\title{
Experimental studies of animal social learning in the wild: Trying to untangle the mystery of human culture
}

\author{
KIM HILL \\ Arizona State University, Tempe, Arizona
}

\begin{abstract}
Here I discuss how studies on animal social learning may help us understand human culture. It is an evolutionary truism that complex biological adaptations always evolve from less complex but related adaptations, but occasionally evolutionary transitions lead to major biological changes whose end products are difficult to anticipate. Language-based cumulative adaptive culture in humans may represent an evolutionary transition of this type. Most of the social learning observed in animals (and even plants) may be due to mechanisms that cannot produce cumulative cultural adaptations. Likewise, much of the critical content of socially transmitted human culture seems to show no parallel in nonhuman species. Thus, with regard to the uniquely human extent and quality of culture, we are forced to ask: Are other species only a few small steps away from this transition, or do they lack multiple critical features that make us the only truly cultural species? Only future research into animal social learning can answer these questions.
\end{abstract}

I believe that research on animal social learning can teach us a great deal about cognition in general, as well as the importance of epigenetic inheritance in biological organisms, but for this article I will focus mainly on the topic of how studies on animal social learning can help us understand human culture. Given the current evidence, it seems that we are still at a point where we have many more questions about culture than answers. It is an evolutionary truism that complex biological adaptations always evolve from less complex but related adaptations, but occasionally evolutionary transitions lead to major biological changes whose end products are difficult to anticipate. Some of these landmark changes include the origins of multicellularity, eukaryotes, chromosomes, sexual reproduction, and perhaps also the cumulative culture that is the main adaptive system of Homo sapiens.

Humans have relied on cumulative culture and language for about the past 100,000 years to become the dominant life form on planet Earth. How we evolved these features from ape species with less-developed capacities of social learning and communication is now a critical scientific mystery (Hill, Barton, \& Hurtado, 2009). Whatever the cause of this transition, genome analysis suggests that human dependence on culture has led to massive recent selection on the genome (Laland, 2008). Language-based information transfer has produced such an astounding divide between us and other organisms that some have proposed it as constituting the most recent major evolutionary transition of life on the planet (Maynard Smith \& Szathmáry, 1995). As with other evolutionary transitions, few intermediate forms are available for study, and emergent properties do not necessarily show a continuum with preadaptations. If these assessments are correct, the fact that all species of hominins but one have disappeared from the planet may be terribly unfortunate for modern researchers. The best we can do is study other animals, especially apes, and hope that they provide some insights into the capacities that have been elaborated in our own phylogenetic line (see, e.g., Whiten, 2010).

Extrapolating from animal social learning to human culture may be a bit like examining a paper glider to guess how a space shuttle is built. Although we can imagine and demonstrate some of the requirements that would turn a glider into a space shuttle - such as an onboard system of propulsion, controls for adjusting lift, and a steering mechanism - speculation about what selective forces could put them in place and what other requirements would convert a glider into a vehicle for space exploration would be complicated. Some changes might not be anticipated by simply extrapolating from more elaborate gliders. For example, a scientist researching the properties of gliders might have never imagined that a highly propelled version of a glider could escape the clutches of gravity and enter a weightless realm in which different properties of flight applied and gyroscope-based navigation was essential.

In this essay, I consider how experimental studies of animal social learning in the wild can help us understand the evolution of human culture. Although others have focused on specific components of human cultural capacity and the possible differences between humans and other ani- 
mals, here I suggest that an increased understanding of the evolution of human culture can best be accomplished by an expansion of research in five topical areas: (1) What are the precise mechanisms employed to learn from conspecifics in large-brained species and species that are highly cooperative? How do these mechanisms differ from those that produce social learning in the vast majority of other species? Is high-fidelity imitation critical, and to what extent does the assumption of cooperative intent between model and learner change the likely character of imitation? (2) What role does capacity for innovation play in the dichotomy between animal traditions and cumulative human culture? (3) Do demographic factors play a critical role in limiting the ability of animals to evolve cumulative improvement in socially learned traditions? Are the networks of significant social interaction notably larger in humans than in other species with well-developed imitative capacity, and does this result in significantly higher rates of exposure to useful rare innovations that could favor the evolution of costly social-learning mechanisms? (4) What are the factors that limit the range of content transmitted by social learning in animals? Many of the spectacular features of human culture seem to derive from languagebased causal understandings of the world and the creation of social norms that regulate competition and cooperation in large groups. Is language critical for the transmission of social norms and increasingly complex understandings of causality? (5) Do animals exhibit mechanisms that can result in cultural group selection of functional traits? Humans have frequently solved cooperative dilemmas by evolving group-beneficial cultural traits (institutions) that override the tendency to pursue individual selfish gain. It appears that these institutions can spread through cultural group selection, yet this process may be absent from all other species.

\section{Social Learning and Mechanisms}

Social learning is often defined as "learning that is influenced by observation of, or interaction with, another animal (usually a conspecific) or its products" (Heyes, 1994, p. 207). Simply put, this means changes in behavioral response after observation of a living model (often conspecific) or its effects upon the environment, where observation can be achieved through any of the perceptive senses (not just vision). By this definition, a wide variety of cognitive abilities and mechanisms, including phenotypic plasticity, can result in "social learning." Some plants change their behavior after sensing herbivore-induced plant volatiles (HIPVs), chemicals emitted from other plants in response to herbivore predation (Choh, Kugimiya, \& Takabayashi, 2006). These plants "learn" from conspecifics that predators are nearby, and as a result they mount various clever preemptive defenses. There are other examples of potential social learning in plants as well. Plants that are shaken by wind action on the branches of other plants may "learn" indirectly from their neighbors that wind is likely to affect them as they grow, and thus adjust their growth patterns appropriately (Berthier \& Stokes, 2005). Further extending the analogies with animals, there is evidence that plants may learn preferentially from close kin
(Karban \& Shiojiri, 2009). Some of these scenarios are still speculative, but the likelihood of plant "social learning" is increasingly apparent as we learn more about plant "intelligence" (Trewavas, 2003). The point here is that "social learning," as currently defined, is not always produced by identical mechanisms, nor are the various types of social learning necessarily relevant to understanding the emergence of evolving adaptive culture.

Since most organisms are capable of observing the environment and using cues of food, predators, and so on, in order to subsequently modify their behavior, we expect that natural selection will usually endow them with the ability to learn from other organisms as well. All living organisms, including conspecifics, can be perceived as stimuli associated with rewards and punishments. If conspecifics are associated with stimuli that themselves have been associated with rewards and punishments, we get second-order conditioning. This is clearly social learning, since new information is obtained and behavior is modified after observing conspecifics. Cadis fly larva, for example, learn to avoid specific fish predators, and bees learn to forage on certain colored flowers, by watching conspecific behavior (Leadbeater \& Chittka, 2007). This type of social learning requires no mechanisms beyond those of individual associative learning. Importantly, second-order conditioning, as well as local and stimulus enhancement that rely on conditioning combined with individual trial-and-error learning, may account for a good deal more social learning in the world than do emulation, imitation, and teaching (Galef, 1992).

Given the ubiquity of second-order conditioning, experiments that purport to contrast species that do and do not engage in social learning (Reader \& Biro, 2010) may simply inform us about individual learning mechanisms. These examples may illustrate, for example, the inability to associate unnaturally paired stimuli through individual learning, just as Garcia's experiments with rats illustrated in the 1960s (Garcia \& Ervin, 1968). If this is true, the methodological concern over unambiguously demonstrating social learning is misplaced. Social learning should be commonplace with the appropriate kinds of stimuli, and the demonstration that a species succeeds or fails in a specific experiment simply tells us about the natural relevance of the experimental conditions. This kind of social learning has been suggested for tens of thousands of species (Lumsden \& Wilson, 1981), and we probably should not be surprised to discover social learning in virtually all species capable of individual learning. A more important biological question, however, is whether these types of social learning mechanisms could lead to behaviors that would never be discovered without exposure to a model (such as most of human culture), and whether these mechanisms could ever lead to adaptive cultural evolution.

Because of the multiple means to achieving "social learning," we are compelled to consider specific mechanisms of learning in order to determine the relevance of observed social learning in species $\mathrm{X}$ for understanding social learning in species Y. Second-order conditioning and enhancement learning do not indicate any cognitive mechanisms beyond those present in all organisms that learn 
through association. Emulation and imitation, on the other hand, require the ability to learn by watching a model's actions and resultant rewards that can be obtained by fulfilling certain requirements, or copying the precise actions of a model applied in a particular context in order to obtain a reward. Although there is much debate over the frequency of true emulation and imitation in animals and their importance for generating cumulative culture (e.g., Byrne, 2005; Heyes, 1996; Shea, 2009; Tomasello \& Carpenter, 2005; Whiten, McGuigan, Marshall-Pescini, \& Hopper, 2009), I suspect that we must focus on these types of social learning if we are to explain the aspects of human culture that make us a uniquely successful species. In some organisms social learning may even include the transfer of components like hierarchical action structure (Whiten, Flynn, Brown, \& Lee, 2006), conceptual rules (Williamson, Jaswal, \& Meltzoff, 2010), and deeper causal principles, inferred by observing a model. The cognitive mechanisms of emulation, imitation, and causal inference based on observing model behavior are likely to be very different from those required to produce second-order conditioning and enhancement learning, and these different mechanisms probably lead to very different developments when the conditions allowing cultural evolution are present.

Researchers in the field of social learning have long been aware that organisms engaging in individual associative learning while observing conspecifics can produce the illusion of true social learning (Galef, 1992). Since secondorder conditioning is readily observed among insects, we can be fairly certain that similar learning frequently takes place among higher primates. Thus, even in primate experiments we should be careful not to jump to conclusions about advanced social learning capacity. Second-order conditioning may potentially account for observations such as attempting to open the same side of a box that a model opened (micro local enhancement) and messing with one set of knobs, switches, or levers and not another set, because a model did so. Evidence of emulation, imitation, and causal inference therefore requires observations of highly improbable actions that demonstrate more than just intensive focus on location or object. Evidence of imitation and causal learning from behavioral observation are evident, for example, when we watch an Eskimo go through the steps of building a kayak. Kayaks cannot be constructed by simply manipulating the same set of materials in the same place as a model has built a kayak. Simply fiddling with the same side of a food-dispensing apparatus does not meet stringent criteria of imitative learning, nor do easily learned natural behaviors, such as probing with a stick. Such behaviors are easily attained using second-order conditioning and some individual trial and error. Thus, designing naturalistic experiments that truly examine how often social learning is based on observing actions and inferring causality from observation (prior to any trial and error learning) will bring us much closer to understanding the gap between human and nonhuman social learning.

\section{Cumulative Cultural Evolution}

The single most notable difference between animals and humans is the ability of humans to rely on cultural evolution as a rapid extragenetic mechanism of adaptation (see Caldwell \& Millen, 2010, in this issue). For adaptive cultural traits to build cumulatively through time, as genetically transmitted phenotypes do, new useful variants must be introduced at a rate higher than they are lost; new variants must spread and "stick" in a population until some modification can be introduced; and useful variants must be spread preferentially, and nonuseful variants must be filtered out (J. Henrich \& McElreath, 2003, 2007).

The process of attaining increasing cultural complexity is mathematically analogous to the process that determines species biodiversity on islands (MacArthur \& Wilson, 1967). New cultural variants can arise through in-situ innovation (mutation) or by introduction (migration) from neighboring populations. If populations are small, the population rate of in-situ innovation will be low (innovation takes place at a rate of $\mu$ per individual per generation, so more individuals in a population simply increases the number of innovations introduced each generation). If populations are distant or socially isolated, new "migrant" types are introduced at a low rate. Likewise, if populations are small, the probability of a stochastic event that eliminates all individuals carrying one variant type is much higher. If the probability of death before reproduction for an individual is $y(0<y<1)$, the rate of independent extinction of all individuals carrying that variant is $y^{n}$, where $n$ is the number of trait carriers. This probability becomes very small if a trait has spread through a large population, so stochastic extinction of a common variant is rare in large populations. Finally, for change to be useful rather than random, there must be an effective filtering process that retains advantageous traits. In genetic evolution this is natural selection, but cultural evolution includes both selection against cultural variants leading to low survival or fertility and biased transmission of variants that are successful. Because imitation of traits can take place many times per generation, and single individuals can be the model for thousands of imitators, biased transmission is often a much more powerful filter on cultural traits than is natural selection.

In sum, large populations that are well connected to other nearby populations can evolve cultural adaptations at a faster pace than smaller, isolated populations can because new useful innovations are introduced at a faster rate (to the population) and, after they have had a chance to spread, are less likely to be eliminated through stochastic processes. If individuals have evolved a cognitive preference for success-biased transmission, highly adaptive traits can rapidly accumulate in large, networked populations. Rapid cultural evolution in large, networked populations is analogous to faster genetic evolution in the modern world (Cochran \& Harpending, 2009).

Experimental studies on animal social learning should be designed to examine each of the components that lead to accumulation of adaptive culture in humans. Specifically animal researchers should examine cognitive mechanisms and demographic factors that affect (1) intrapopulation innovation rates, (2) rates of interpopulation spread of innovations developed elsewhere, (3) spread and retention of variants through mechanisms of high-fidelity transmis- 
sion, and (4) filtering of nonadaptive variants through natural selection, biased transmission, and cultural group selection.

\section{Areas of Fruitful Research}

To understand the emergence of human culture, research should focus on five areas using naturalistic experiments with animals. First, we need to know more about the mechanisms that are critical for cultural evolution. Imitation seems to allow for rapid, high-fidelity transmission of innovation. Human children regularly engage in "unrewarded imitation" and expend time practicing socially learned traits so that their performance will be superior when an opportunity for expression arises. It would be useful to determine, using natural experiments, how many other species "practice" behaviors that are socially learned when no short-term payoff is possible. This appears to be an indicator of imitation rather than enhancement or second-order conditioning. Naturalistic observations to detect imitation must be complemented with experimental methods such as "ghost displays" and "end-state demonstrations" (Hopper, in press), which can probably be transferred from laboratory to wild settings. We also need to carefully determine whether alternative cognitive mechanisms may produce imitation in different animals; for example, is the reported tendency of human children to "overimitate" relative to chimpanzees due to peculiar learning mechanisms (Whiten et al., 2009) or to a more general capacity for imitation combined with a strong tendency in humans to conform to social norms?

Transmission biases based on the success or frequency of variants and on the characteristics of observed models also seem to play a major part in the adaptive nature of human cultural patterns (Boyd \& Richerson, 1985). For example, success-biased transmission appears critical for ratcheting up cultural effectiveness (J. Henrich, 2004b), and conformity bias may be critical for maintaining stable group equilibria that allow for cultural group selection (Boyd \& Richerson, in press; J. Henrich, 2004a). In addition, cultural evolution requires horizontal transmission within and/or between groups, not just vertical transmission from parents to offspring. Thus, impressive toolusing species, such as New Caledonian crows, that appear to engage almost exclusively in parent-to-offspring transmission (Holzhaider, Hunt, \& Gray, 2010) are unlikely to generate extensive cultural ratcheting.

It is also important to note that transmission biases can differ significantly even when outcomes appear to be similar. For example, both humans and animals may show "cultural phylogenies" (Lycett, 2010), yet multiple mechanisms can produce this result. Both parent-offspring learning and in-group conformity bias lead to the grouplevel vertical transmission required to produce cultural phylogenies. Thus, it is important to develop methods to detect the mechanism of transmission, even when outcomes appear identical.

Other questions about transmission must also be addressed with careful experiments in animals. Do animals ever observe multiple models and then choose to copy the most efficient or the most common? What is the rela- tionship between imitation and cooperation? Does shared intentionality (Tomasello \& Carpenter, 2007) simply enhance some mechanisms of social learning, or does it lead to the evolution of new mechanisms? Do animals imitate more precisely when they assume that their behavioral model is less motivated to deceive them or to exploit the learning process for manipulation? Is imitation more effective between kin and within cooperative breeding species? Is teaching rare only because unrelated models are not motivated to provide honest information to observers/ learners (see Thornton \& Raihani, 2010)? Can we expand experimental studies of reciprocity in animals to include opportunities for teaching among dyads that engage in reciprocity in other realms?

The second area of useful research concerns the nature of innovation, which has been investigated in some animal species but is still poorly documented for many (Reader $\&$ Laland, 2003). Innovation rates for previously socially learned behavior seem extraordinarily high among humans relative to other animals. Is this impression correct? For example, I suspect that an adult human, taught to fish for termites with twigs, would develop significant improvements in technique and technology within a few hours. Yet the spread of behaviors in wild animals is difficult to observe in part because innovations are so rare. We also need more information concerning the difference between random learning errors and "guided variation" (Richerson \& Boyd, 2005, p. 116), in which modifications on socially learned behaviors are usually improvements. Guided variation allows for much more rapid cultural evolution.

Although long-term natural habitats may offer few chances to invent something not already discovered in past generations, the novel environments associated with migration, displacement, and captivity should provide plenty of opportunities for animal innovation. We need systematic data on rates of innovation under such circumstances. We also need better quantitative naturalistic data on innovation in small-scale human societies to complement laboratory studies such as those reported here by Caldwell and Millen (2010).

One observation may be critical to understanding the high rate of human innovation. Empirical studies show that groups of individuals working together are more likely to discover innovative solutions than are even the best individual innovators in any group (Laughlin, Hatch, Silver, \& Boh, 2006). This is probably why academic and business research units generally work in teams. Thus, humans may innovate at higher rates than apes because of social interaction patterns. High levels of cooperative interaction among human foragers (see, e.g., Hill, 2002) may lead to innovative foraging techniques and technology much faster than can be achieved by ape species that mainly forage alone throughout the day. Experiments on innovation in foraging traditions should thus concentrate especially on animal species that forage as a group. A similar human advantage may have arisen via bipedalism and increased manual dexterity. We should examine the extent to which manipulative dexterity is a better predictor than intelligence of tool innovation in species that manufacture tools. Continuing this line of reasoning, we also need in- 
formation on the extent to which language is a critical determinant of the human ability to produce guided variation in much shorter time periods than are required for other animals. Such research is complicated by the fact that few species can communicate effectively in ways relevant to innovation, but experimental work with humans in which communication is limited could easily measure the impact of reduced communication on rates of innovation.

A third area of potentially fruitful research concerns demography and social network size. Theoretical models show that social interactant network size is critical to the accumulation of improvements on social traditions (e.g., J. Henrich, 2004b). Humans have evolved an exceptional social structure that allows many hundreds to many thousands of individuals regular exposure to each other's innovations, even in hunter-gatherer societies (Hill et al., 2010). The human social structure, as manifest in hunter-gatherer societies, is based on pair bonding and paternal investment in multimale-multifemale groups, with reciprocal mate giving and frequent friendly association between affines across multiple residential groups (Chapais, 2008; Hill et al., 2010). This social structure allows new behavioral variants to be rapidly introduced from a large population of potential innovators on the basis of frequent face-to-face interaction between individual members of social units (ethnolinguistic tribes) that are generally at least two orders of magnitude larger than chimpanzee communities (Binford, 2001, pp. 118-129). This huge difference in the rate of potential exposure to novel behaviors could explain why humans have evolved costly social-learning mechanisms not present in other species (Boyd \& Richerson, 1996). Notably, in human societies there is significant between-group transfer of fully grown adults of both sexes, not just juveniles (who are less likely to have mastered the cultural traditions of a particular group). Therefore, field experiments on nonhumans should specifically focus on the opportunities for intergroup transfer of behavioral traditions beyond occasional between-group migration.

One way to examine demographic effects on cumulative culture is to simulate rapid introduction of innovations via experimental seeding in subgroups. If a group of animals is divided, and one subgroup is seeded with the first step of a useful process, while the other subgroup is seeded with the second step, can individuals in the two subgroups quickly learn to complete the two-step process when reunited? If so, this might suggest that larger network size is a critical feature whose lack retards accumulation in animal culture.

A fourth area of productive research is experiments designed to look for a wider range of content that can be transferred through animal social learning. Do animals socially learn and transmit unverified beliefs, false beliefs, rules or social norms, signals of such rules or norms, or any signals other than those of local recognition? Do animals transmit flexible aspects of mating systems or social structure? Do they ever socially learn altruistic or group-beneficial traits? Human children readily imitate altruistic and "other-regarding" behaviors (N. Henrich \& Henrich, 2007, pp. 28-29) and incorporate an emotional reaction against doing things in a way other than the one demonstrated by an adult model (Rakoczy, Warneken, $\&$ Tomasello, 2008). Are there reasons related to sociallearning mechanisms why some of these content categories cannot be transmitted in nonhuman animals?

Finally, we need more experimental work on life history shifts in social-learning patterns. In humans, children sometimes overimitate adult models, but not each other (Flynn \& Whiten, 2010), and it is doubtful whether adults ever knowingly overimitate. Since prestige-biased transmission is a form of overgeneralization during imitation, it would be interesting to determine whether such bias is also primarily expressed in juveniles. We also know little about life history changes in other transmission biases. Does reliance on conformity versus success-biased transmission change through the life course? Is this true in other animals? What does the age profile of imitation accuracy versus modification look like in humans and in other animals? Do juvenile animals show higher-fidelity copying when models are adults, as compared with juvenilejuvenile or adult-adult transmission? In humans, adults engage in social learning through much of their lifespan, but the content of what can be learned seems to change (language, values, and food preferences may be set early, whereas techniques and technologies are often learned in middle adulthood or even later). Is the sensitive period of learning for socially transmitted behaviors restricted in other animals?

Another important life history pattern of human culture is observed in innovation rates. Significant innovations that lead to adaptive cultural evolution in human societies are mainly introduced by young adults. Major breakthroughs in all fields of knowledge tend to come from adults 30-40 years of age (Lehman, 1953). This seems to be driven by the age patterning of "divergent thinking" in general (McCrae, Arenberg, \& Costa, 1987): Children mainly imitate; young adults experiment extensively, modifying behaviors they have learned through imitation; and older adults show reduced capacities of innovation and social-learning skills. Is this pattern universal in primates?

\section{Experimental and Statistical Techniques to Detect Social Learning}

As mentioned above, the expected ubiquity of social learning broadly defined means that the simple detection of social learning provides limited information about human culture. Some of the new methods for detecting social learning are effective but do not distinguish conditioning, enhancement, and imitation, which may be critical to understanding cumulative culture. The Hoppitt, Kandler, Kendal, and Laland (2010) report in this issue presents an example of how methods currently employed might confuse second-order conditioning and imitation. Likewise, the option-bias analysis discussed by Kendal et al. (2010) is based on the improbability of control subjects consistently choosing one of two options available to obtain a reward unless the preference is acquired through social learning. Although this may demonstrate social learning, it does not distinguish between second-order 
conditioning (on a micro scale) and imitation. Likewise, the NBDA method described by Franz and Nunn (2010) is a sophisticated method for determining the probability that behavioral traits have spread along specified social networks, but it cannot distinguish between spread due to second-order conditioning, enhancement, or imitation. It may also fail to detect some types of social learning because of its assumptions about mechanisms; for example, with strongly prestige-biased transmission, a single exposure to a high-prestige model is more likely to lead to transmission than are multiple exposures to a low-prestige model. Since this type of learning will not correlate well with network interaction patterns, the maximum likelihood network criteria would not identify this form of social learning. Despite these reservations, I do believe that the new methods illustrate important avenues for further methodological advances. For example, one could easily modify the maximum likelihood approach developed in NBDA to distinguish individual from social learning so as to also distinguish the likelihood of transmission due to different types of transmission biases and the likelihood of mixes of individual and social learning (see, e.g., Efferson, Lalive, Richerson, McElreath, \& Lubell, 2008; McElreath et al., 2008). No doubt the same statistical methods could be used to compare the behavioral patterns expected from conditioning and enhancement versus emulation and imitation, in order to make probabilistic statements about the mechanisms of social learning employed by different species.

\section{Human Culture Versus Animal Traditions}

I have previously suggested that employing the term culture to describe socially transmitted behavioral traditions in animals may not be scientifically productive. I do not consider this discussion to constitute intellectual warfare, but simply to reflect a concern that our terminology enhance rather than impede understanding.

My concern is that the loose application of the term "culture" for all socially learned behavior may obscure our ability to understand the evolution of what appear to be very unique characteristics of Homo sapiens. If humans are indeed as exceptional as they appear to be, we must describe more accurately their special characteristics rather than eagerly lump them with other social vertebrates. (Hill, 2009, p. 271)

The first definition of culture - proposed by E. B. Tylor (1871, p. 1), the father of anthropology - declared culture to be "that complex whole which includes knowledge, belief, art, law, morals, custom, and any other capabilities and habits acquired by man as a member of society." Although we may quibble with Tylor's assertion that culture is by definition human, he clearly proposed that culture included social norms (law, morals), ethnic marking (art, customs), and ritual (belief, customs). Other widely used anthropological definitions have also emphasized values, beliefs, norms, and rules above all else (Hill, 2009). In other words, the term culture, proposed by anthropologists, has generally consisted of socially learned content of a particular type, not just any socially learned behaviors.
The assertion that "animals have culture" has mainly been embraced through a redefinition of the term culture. I have no desire to argue over semantics, but it is difficult not to be a bit stunned when the researchers in one field of study decide to redefine a key theoretical term developed in another field without consultation or discussion. Redefinition of the word culture by nonanthropologists, absent significant exchange with cultural anthropologists, seems intellectually inappropriate, just as it would if cultural anthropologists were to redefine the term gene without seriously engaging molecular biologists about the issue. However, I also recognize that most cultural anthropologists have opted out of discussion about the possibility of nonhuman culture, and seem to have lost interest in basic theoretical questions about the meaning of the term. Indeed, if current trends continue, they will lose all claim to expertise in their own specialty area within the next generation. I thus find myself in the awkward position of playing devil's advocate for an anthropological viewpoint that I believe represents the views of many cultural anthropologists, but which is also based on my own underlying orientation to evolutionary thinking.

The employment of the term culture to describe animal traditions seems to derive from assumptions about similarities that have yet to be demonstrated. For example, Lonsdorf and Bonnie (2010) state in this issue that

A unifying theory of cultural evolution will necessitate ... the recognition of humans and animals as lying on a continuum of social learning and cultural capacity. (p. 202)

Whether human cultural patterns can be extrapolated from the continuum of social-learning patterns in nonhuman species is an open question, in my opinion. If an extraterrestrial alien scientist were fully informed about the range of social-learning patterns observed in all other animal species on this planet, could that scientist predict that human-like culture would emerge (without observing it), and would he or she anticipate its features? This would be the implication of "lying on a continuum." I have some doubts.

I previously suggested (Hill, 2009) that socially transmitted behavior shared by a group of individuals is more appropriately termed a behavioral tradition, and I believe that it is more accurate to say that animals have behavioral traditions that are the building blocks of culture, rather than culture itself. I note that a fair number of the contributors to this issue seem comfortable with this position as well. Indeed, it seems mainly to be the primatologists who prefer to label their animals and social groups as "cultural." Perhaps they are correct, if imitation is the most important component of culture, but they may be mistaken if social norms are considered more important. Interestingly, although chimpanzee behavioral traditions are the most likely to be called "cultural," social insects may come closer to meeting many anthropological criteria. Social insects learn behaviors that are transmitted across generations, including language dialects that can be learned by outsiders; unrelated individuals co-resident in a social group (multifoundress colony) signal mem- 
bership by smells that are fabricated from chemicals derived from socially learned food preferences (ethnicity); and social insects exhibit incipient norms and third-party punishment, whose character may be socially transmitted (Hölldobler \& Wilson, 2009; Leadbeater \& Chittka, 2007; Moore \& Liebig, 2010). The issue is whether mechanism or content is the critical criterion of culture. I suggest that perhaps both are critical, and that neither chimpanzees nor social insects meet the complete criteria.

According to most anthropological definitions, traditions must be shared by a social group, backed by social norms, reinforced by ritual, and signaled (ethnicity) to constitute culture (Hill, 2009). Social norms are a particularly unique piece of human culture because they often override the ecological costs and benefits of specific behavioral alternatives so as to "determine" optimal behaviors for the members of a particular social group. Social norms in humans are part of emotionally charged belief systems and are generally backed by third-party punishment. This means that individuals who observe norm violations, even when not directly affected by the offender's action, will often punish norm violators, possibly at a significant cost to themselves (J. Henrich et al., 2006; Nelissen, 2008). Humans learn both norms and the appropriate punishments from members of their same cultural group. In humans, competition and conflict over all important resources (mates, territories, food, signaling opportunities, etc.) are regulated by social norms, and collective action problems are often solved by cultural institutions (Hill, 2009). Socially transmitted regulatory norms and institutions appear absent from all other species - with the possible exception of some social insects, who do engage in third-party punishment (Liebig, Peeters, \& Hölldobler, 1999) and show some flexibility in the conditions (norms) that elicit such punishment (Moore \& Liebig, 2010). It is not known, however, whether such norms are sometimes socially transmitted.

Human culture also includes socially learned beliefs that are not directly observable but can be elicited through conversation (something that animals do not do). The existence of these generally invisible beliefs can be detected through behavior, sometimes in situations never before experienced by individuals who have acquired the belief. The transmission of unobserved beliefs may not be possible in animals, who instead either copy or do not copy observed behavioral patterns, and thus produce only similar behaviors in contexts similar to those that were observed in a model. Finally, human culture includes language as a system of communication, but also as the system for most transmission of culture itself. A huge fraction of human cultural content probably cannot be socially learned without language. Thus, I find it puzzling that some researchers suggest that apes effectively bridge the culture-no culture gap with humans, but then concede that there is no bridge of the language-no language gap in nature (e.g., Whiten, 2010). Since language is the means by which critical human cultural content is transmitted, this suggests to me that apes are far from bridging the culture-no culture gap between humans and other animals.
In sum, a set of traditions and beliefs, backed by social norms that enforce them, and including the language and signals designed to ensure their transmission (ritual) and to indicate adherence (ethnicity) constitute "a culture." All human societies have culture (not just behavioral traditions), and all humans normally develop membership in specific cultural groups (sometimes more than one). Many animals exhibit behavioral traditions, and a few species even develop sets of traditions held in common, but no animal species appears to have "a culture" according to the anthropological definition I have outlined above.

Human culture consists of another feature that is absent from animals: Humans develop cultural practices and behavioral traditions that progressively change through time, building on previous patterns to achieve greater utility and complexity. Lycett (2010) suggests that chimpanzee culture is also composed of "multiple traditions forming mosaics of behavioral patterns that collectively evolve via a process of descent with modification." But chimpanzee material "culture" is no more advanced now than it was during the Pleistocene. If specialization into two tool types - digging sticks and brush probes-represents the 5-million-year cultural evolution of termite fishing technology in chimpanzees, then chimpanzee cultural evolution has proceeded at a rate indistinguishable from that expected through individual learning. Not surprisingly, modern chimpanzees raised with humans do not learn any significant amount of human culture, especially not beliefs, morals, and ethnic markers. But human children, raised with chimpanzees, do learn to imitate some chimpanzee behavioral traditions (N. Henrich \& Henrich, 2007, pp. 7-8). The gap between the two species seems tremendous.

The weakness of referring to all socially acquired traits as culture becomes apparent when we consider examples, in human societies, of socially learned traits that would not be considered cultural. First, laypeople and anthropologists do not consider individually expressed traits to be cultural, even if they are acquired through social learning. Culture is something expressed by groups. Thus, a hunter's avoidance of a particular trail because he has observed another hunter getting stung by wasps on that trail is not a cultural trait, even though it is socially learned. Second, traits that are not backed by social norms or used to reinforce norms are not generally considered cultural, even if they are learned by observing a model. Rainforestdwelling hunter-gatherers swim and climb trees. Grassland desert foragers usually do neither, and despite the fact that these behaviors are learned by imitation, this behavioral difference would not be considered by many anthropologists to be cultural. Ache hunter-gatherers do not say "I climb trees because it is my culture," but they do emphatically pronounce that they share food widely "because it is our culture." Cultural traits are adopted because other members of one's social group exhibit those traits, and there is often a socially transmitted belief that to do otherwise is wrong (Rakoczy et al., 2008).

Ultimately, the use of the term culture to describe animal behavioral traditions may become standard among researchers. If so, I too will comply, since language is 
nothing more than the use of arbitrary symbols for conventionally agreed-upon meanings. But I suspect this will then force us to repeatedly use modifiers (e.g., "languagebased culture backed by social norms and advertised by signaling") whenever we refer to human culture and wish to contrast it with the "culture" of animals. If so, the redefinition may represent a bit of semantic sabotage rather than a useful step forward.

\section{The Importance of Language in Human Cultural Evolution}

Maynard Smith and Szathmáry (1995) have suggested that human language is the last major transition in evolution on Earth. Language is itself socially learned and is subject to modification due to transmission biases that tend to give it properties that make it easily learned by simple exposure during childhood (Smith \& Kirby, 2008). But language also has a profound effect on human cultural evolution. Because much social learning in humans is carried out through teaching, language facilitates extremely complex and high-fidelity learning that would be done much less efficiently through observation alone. For example, it is much more difficult to learn how to correctly build a complex composite tool if one relies entirely on observation, without any verbal instruction. Even more importantly, language allows for the social transmission of the unobservable. This means that conceptual models of causality can be transmitted and built upon through time to achieve deep understandings of likely relationships that then have practical applications to behavior and to subsequent innovation on alreadyexisting cultural traits. This ability to transmit complex abstractions is probably unique to humans. Informational areas like theoretical physics allow for tremendous cumulative evolution of concepts prior to any observable expression in behavior. Likewise, language permits conceptual norms of behavior that can be transmitted effectively, with clear understandings about what constitutes a defection from the norm and what are the agreed-upon social consequences. This ability may be critical to understanding how humans alone have achieved extensive cooperation in large groups of non-kin.

Language, unique to humans and no other species, seems critical for the magnitude of cultural ratcheting that has taken place in the past few tens of thousands of years in our species. Language has facilitated improvements upon itself as a means of information transmission - from speaking, to writing, to printing, to high-speed copying, and finally to digitally encoded information transfer. These innovations have broken the demographic constraints of direct social interaction as a source of model behavior and have allowed humans to take advantage of innovations discovered by millions of individuals never observed, who may in fact be long dead. The outcome of this process has been to network together hundreds of millions of individuals who can be exposed to the innovations and unobservable concepts developed by distant peers and past generations, and to ratchet up cultural complexity in an increasing spiral that shows no signs of slowing down. My Ache hunter-gatherer friends, who went through child- hood exposed to a toolkit of less than 25 items, illustrate the universal human ability to tap into language-based information transfer. Within a single lifetime, they have gone from using bone chisels and stone axes to iPods, cell phones, GPS receivers, and laptops. It is no wonder that humans dominate the planet biologically and appear to be so qualitatively different from any other species. Natural and experimental research on conditions that lead to complex communication systems is probably essential to understanding the gap between human culture and nonhuman behavioral traditions.

\section{Two Types of Cultural Evolution}

Much has been made of the ratchet effect and the fact that animals do not show cumulative cultural evolution. To build adaptive complexity through social learning, the modification process must be combined with a filter, such as success-biased imitation, so that modifications are increasingly more functional rather than random. Indeed, success-biased copying can produce adaptive culture because of the effects of individual advantage. But even if some examples of this type of adaptive cumulative social learning are empirically verified in animals, humans still exhibit another type of cultural evolution that may be impossible in other species. This form of cultural evolution produces group-beneficial cultural adaptations (i.e., institutions) rather than behavioral traditions that result in higher individual success at evolved goals. Theoretical models show that when culturally structured populations maintain between-group cultural variation due to social norm enforcement or conformity bias (J. Henrich \& Boyd, 2001), selection can take place between different cultural equilibria (Boyd \& Richerson, 2002, in press; J. Henrich, 2004a). Equilibrium cultural traits that promote group welfare, such as particularly efficient social structures, cooperative economic institutions, norms of self-sacrifice in warfare, and so on, can spread at the expense of individually beneficial behaviors that decrease overall group welfare. In short, altruistic behaviors can spread via their effects on group success. The spread of group-beneficial culture can be accomplished through direct violent confrontation between groups (see, e.g., Soltis, Boyd, \& Richerson, 1995), through the incorporation of migrants into the cooperative societies that are more attractive because of the greater success of their members, and through rapid intergroup copying of more successful behaviors (Boyd \& Richerson, in press). Such cultural group selection has likely been common among humans in the past few tens of thousands of years, but it relies on mechanisms that make conformity to the cooperative norm more likely than selfish behavior. Unlike genetic group selection, cultural group selection is not undermined by migration, as long as social norms or conformity bias keep groups at different cultural equilibria. Is this process possible in animals? Some animals do show conformity-biased transmission (Lonsdorf \& Bonnie, 2010), but it is unclear whether this can maintain group-level differences in the face of frequent migration. If so, perhaps cultural group selection in animals might be observed as well. 


\section{Conclusion}

A careful comparison of the use and consequences of social learning in humans and other animals suggests a huge gap. Culture can be an effective adaptive system, but this requires cumulative cultural evolution through individual and/or group selection on cultural traits. At present, neither type of cultural evolution seems to take place at any appreciable rate in animals. Ironically, species with intermediate levels of cultural capacity were probably exterminated by our own more successful ancestors as they became fully committed to cultural adaptation.

What we can now learn from animal experiments will depend on paying careful attention to some of the apparently critical properties of human social learning that result in the unique human outcome. Some animals are capable of true imitative learning and pass behavioral traditions intergenerationally, but they do not transmit cultural content. Social insects, on the other hand, have developed simple language and social norms, and they signal group membership in genetically mixed colonies, but intergenerational transmission is often due to epigenetic transmission rather than imitative learning. The mechanisms and content of social learning both seem to be important for understanding how humans evolved culture; thus, we must study a variety of species to understand how these factors might interact.

Finally, the capacity for high-fidelity imitation of conceptual as well as behavioral information, in conjunction with the highly cooperative nature of our species' life history, led to language. Language then facilitated new levels of information transmission unparalleled in the history of life on earth, which have subsequently allowed social learning to engulf all aspects of human existence. Are other species only a few small steps away from this transition, or do they lack multiple critical features that make us alone the truly cultural species? Only future research into animal social learning can answer these questions.

\section{AUTHOR NOTE}

Correspondence related to this article may be sent to K. Hill, School of Human Evolution and Social Change, Institute of Human Origins, Arizona State University, SHESC 233, P.O. Box 872402, Tempe, AZ 85287-2402 (e-mail: kim.hill@asu.edu).

\section{REFERENCES}

Berthier, S., \& Stokes, A. (2005). Phototropic response induced by wind loading in Maritime pine seedlings (Pinus pinaster Aït.). Journal of Experimental Botany, 56, 851-856.

BINFORD, L. R. (2001). Constructing frames of reference: An analytical method for archaeological theory building using hunter-gatherer and environmental data sets. Berkeley: University of California Press.

BOYd, R., \& Richerson, P. J. (1985). Culture and the evolutionary process. Chicago: University of Chicago Press.

Boyd, R., \& Richerson, P. J. (1996). Why culture is common but cultural evolution is rare. Proceedings of the British Academy, 88, 77-93.

Boyd, R., \& Richerson, P. J. (2002). Group beneficial norms can spread rapidly in a structured population. Journal of Theoretical Biology, 215, 287-296.

BOYD, R., \& Richerson, P. J. (in press). Transmission coupling mechanisms: Cultural group selection. Philosophical Transactions of the Royal Society $B$

BYRNE, R. W. (2005). Detecting, understanding, and explaining imitation by animals. In S. Hurley \& N. Chater (Eds.), Perspectives on imitation: From neuroscience to social science (Vol. 1, pp. 225-242). Cambridge, MA: MIT Press.

Caldwell, C. A., \& Millen, A. E. (2010). Human cumulative culture in the laboratory: Effects of (micro) population size. Learning \& Behavior, 38, 310-318.

Chapais, B. (2008). Primeval kinship: How pair-bonding gave birth to human society. Cambridge, MA: Harvard University Press.

Снон, Y., KugimiYa, S., \& TAKabaYAShi, J. (2006). Induced production of extrafloral nectar in intact lima bean plants in response to volatiles from spider mite-infested conspecific plants as a possible indirect defense against spider mites. Oecologia, 147, 455-460.

COCHRAN, G., \& HARPENDING, H. (2009). The 10,000 year explosion: How civilization accelerated human evolution. New York: Basic Books.

Efferson, C., Lalive, R., Richerson, P. J., McElreath, R., \& LuBELL, M. (2008). Conformists and mavericks: The empirics of frequency-dependent cultural transmission. Evolution \& Human Behavior, 29, 56-64.

FlynN, E., \& Whiten, A. (2010). Studying children's social learning experimentally "in the wild." Learning \& Behavior, 38, 284-296.

FranZ, M., \& NUNN, C. L. (2010). Investigating the impact of observation errors on the statistical performance of network-based diffusion analysis. Learning \& Behavior, 38, 235-242.

GALEF, B. G., JR. (1992). The question of animal culture. Human Nature, 3, $157-178$.

GARCIA, J., \& ERVIN, F. R. (1968). Gustatory-visceral and telereceptorcutaneous conditioning: Adaptation in internal and external milieus. Communications in Behavioral Biology, 1, 389-415.

HENRICH, J. (2004a). Cultural group selection, coevolutionary processes and large-scale cooperation. Journal of Economic Behavior \& Organization, 53, 3-35.

HenRICH, J. (2004b). Demography and cultural evolution: How adaptive cultural processes can produce maladaptive losses-The Tasmanian case. American Antiquity, 69, 197-214.

Henrich, J., \& Boyd, R. (2001). Why people punish defectors: Weak conformist transmission can stabilize costly enforcement of norms in cooperative dilemmas. Journal of Theoretical Biology, 208, 79-89.

Henrich, J., \& McElreath, R. (2003). The evolution of cultural evolution. Evolutionary Anthropology, 12, 123-135.

Henrich, J., \& McElreath, R. (2007). Dual inheritance theory: The evolution of human cultural capacities and cultural evolution. In R. I. M. Dunbar \& L. Barrett (Eds.), Oxford handbook of evolutionary psychology (pp. 555-570). Oxford: Oxford University Press.

Henrich, J., McElreath, R., Barr, A., Ensminger, J., Barrett, C., BOLYANATZ, A., ET AL. (2006). Costly punishment across human societies. Science, 312, 1767-1770.

HENRICH, N., \& HeNRICH, J. (2007). Why humans cooperate: A cultural and evolutionary explanation. Oxford: Oxford University Press.

HeYes, C. M. (1994). Social learning in animals: Categories and mechanisms. Biological Reviews, 69, 207-231

HeYes, C. M. (1996). Identifying and defining imitation. In C. M. Heyes \& B. G. Galef, Jr. (Eds.), Social learning in animals: The roots of culture (pp. 211-220). San Diego: Academic Press.

HILL, K. (2002). Altruistic cooperation during foraging by the Ache, and the evolved human predisposition to cooperate. Human Nature, 13, 105-128.

HILL, K. (2009). Animal "culture"? In K. N. Laland \& B. G. Galef(Eds.), The question of animal culture (pp. 269-287). Cambridge, MA: Harvard University Press.

Hill, K., Barton, M., \& Hurtado, A. M. (2009). The emergence of human uniqueness: Characters underlying behavioral modernity. Evolutionary Anthropology, 18, 187-200.

Hill, K., Walker, R., Bozicevic, M., Eder, J., Headland, T., HewLETT, B., ET AL. (2010). Kinship coresidence patterns in huntergatherer societies. Manuscript under review.

Hölldobler, B., \& Wilson, E. O. (2009). The superorganism: The beauty, elegance, and strangeness of insect societies. New York: Norton.

Holzhaider, J. C., Hunt, G. R., \& Gray, R. D. (2010). Social learning in New Caledonian crows. Learning \& Behavior, 38, 206-219.

Hopper, L. M. (in press). "Ghost" experiments and the dissection of social learning in humans and animals. Biological Reviews. doi:10.1111/j.1469-185X.2010.00120.x 
Hoppitt, W., Kandler, A., Kendal, J. R., \& Laland, K. N. (2010). The effect of task structure on diffusion dynamics: Implications for diffusion curve and network-based analyses. Learning \& Behavior, 38, 243-251.

KARBAN, R., \& SHIOJIRI, K. (2009). Self-recognition affects plant communication and defense. Ecology Letters, 12, 502-506.

Kendal, R. L., Custance, D. M., Kendal, J. R., Vale, G., Stoinski, T. S., Rakotomalala, N. L., \& Rasamimanana, H. (2010). Evidence for social learning in wild lemurs (Lemur catta). Learning \& Behavior, 38, 220-234.

LALAND, K. N. (2008). Exploring gene-culture interactions: Insights from handedness, sexual selection and niche-construction case studies. Philosophical Transactions of the Royal Society B, 363, 3577 3589.

Laughlin, P. R., Hatch, E. C., Silver, J. S., \& Boh, L. (2006). Groups perform better than the best individuals on letters-to-numbers problems: Effects of group size. Journal of Personality \& Social Psychology, 90, 644-651.

Leadbeater, E., \& ChittKa, L. (2007). Social learning in insectsFrom miniature brains to consensus building. Current Biology, 17, R703-R713.

Lehman, H. C. (1953). Age and achievement. Princeton, NJ: Princeton University Press.

Liebig, J., Peeters, C., \& Hölldobler, B. (1999). Worker policing limits the number of reproductives in a ponerine ant. Proceedings of the Royal Society B, 266, 1865-1870.

Lonsdorf, E. V., \& Bonnie, K. E. (2010). Opportunities and constraints when studying social learning: Developmental approaches and social factors. Learning \& Behavior, 38, 195-205.

Lumsden, C. J., \& Wilson, E. O. (1981). Genes, mind, and culture: The coevolutionary process. Cambridge, MA: Harvard University Press.

LyCETT, S. J. (2010). The importance of history in definitions of "culture": Implications from phylogenetic approaches to the study of social learning in chimpanzees. Learning \& Behavior, 38, 252-264.

MacArthur, R. H., \& Wilson, E. O. (1967). The theory of island biogeography. Princeton, NJ: Princeton University Press.

Maynard Smith, J., \& Szathmáry, E. (1995). The major transitions in evolution. Oxford: W. H. Freeman Spektrum.

McCrae, R. R., Arenberg, D., \& Costa, P. T., Jr. (1987). Declines in divergent thinking with age: Cross-sectional, longitudinal, and crosssequential analyses. Psychology \& Aging, 2, 130-137.

McElreath, R., Bell, A. V., Efferson, C., Lubell, M., Richerson, P. J., \& WARING, T. (2008). Beyond existence and aiming outside the laboratory: Estimating frequency-dependent and pay-off-biased social learning strategies. Philosophical Transactions of the Royal Society B, 363, 3515-3528.

Moore, D., \& LIEBIG, J. (2010). Social regulation changes with colony growth in an ant. Unpublished manuscript, Arizona State University, School of Life Sciences.
Nelissen, R. M. A. (2008). The price you pay: Cost-dependent reputation effects of altruistic punishment. Evolution \& Human Behavior, 29, 242-248.

Rakoczy, H., Warneken, F., \& Tomasello, M. (2008). The sources of normativity: Young children's awareness of the normative structure of games. Developmental Psychology, 44, 875-881.

REAdER, S. M., \& Biro, D. (2010). Experimental identification of social learning in wild animals. Learning \& Behavior, 38, 265-283.

Reader, S. M., \& Laland, K. N. (2003). Animal innovation. Oxford: Oxford University Press.

Richerson, P. J., \& Boyd, R. (2005). Not by genes alone: How culture transformed human evolution. Chicago: University of Chicago Press.

SheA, N. (2009). Imitation as an inheritance system. Philosophical Transactions of the Royal Society B, 364, 2429-2443.

Smith, K., \& KIRBY, S. (2008). Cultural evolution: Implications for understanding the human language faculty and its evolution. Philosophical Transactions of the Royal Society B, 363, 3591-3603.

Soltis, J., Boyd, R., \& Richerson, P. J. (1995). Can group-functional behaviors evolve by cultural group selection? An empirical test. Current Anthropology, 36, 473-494.

Thornton, A., \& RaiHAnI, N. J. (2010). Identifying teaching in wild animals. Learning \& Behavior, 38, 297-309.

Tomasello, M., \& CARPenter, M. (2005). Intention reading and imitative learning. In S. Hurley \& N. Chater (Eds.), Perspectives on imitation: From neuroscience to social science (pp. 133-148). Cambridge, MA: MIT Press.

Tomasello, M., \& CARPenter, M. (2007). Shared intentionality. Developmental Science, 10, 121-125.

Trewavas, A. (2003). Aspects of plant intelligence. Annals of Botany, 92, $1-20$.

Tylor, E. B. (1871). Primitive culture. London: Murray.

Whitehead, H. (2010). Conserving and managing animals that learn socially and share cultures. Learning \& Behavior, 38, 329-336.

Whiten, A. (2010). Ape behavior and the origins of human culture. In P. M. Kappeler \& J. B. Silk (Eds.), Mind the gap: Tracing the origins of human universals (pp. 429-450). Heidelberg: Springer.

Whiten, A., Flynn, E., Brown, K., \& Lee, T. (2006). Imitation of hierarchical action structure by young children. Developmental Science, 9, 574-582.

Whiten, A., McGuigan, N., Marshall-Pescini, S., \& Hopper, L. M. (2009). Emulation, imitation, over-imitation and the scope of culture for child and chimpanzee. Philosophical Transactions of the Royal Society $B, 364,2417-2428$.

Williamson, R. A., Jaswal, V. K., \& Meltzoff, A. N. (2010). Learning the rules: Observation and imitation of a sorting strategy by 36-month-old children. Developmental Psychology, 46, 57-65.

(Manuscript received May 7, 2010; accepted for publication May 10, 2010.) 